\title{
Circ_0000527 promotes the progression of retinoblastoma by regulating miR-646/LRP6 axis
}

\author{
Li Zhang ${ }^{1}$, Jie Wu' ${ }^{1}$, Yujun Li ${ }^{1}$, Yanxia Jiang ${ }^{1}$, Lili Wang ${ }^{1}$, Yunqing Chen ${ }^{1}$, Yalin Lv²* ${ }^{*}$, Yuwei Zou ${ }^{1}$ \\ and Xiaoyan Ding ${ }^{1}$
}

\begin{abstract}
Background: Researches validate that circular RNAs (circRNAs) are dysregulated in a variety of malignancies and play an important role in regulating the malignant phenotype of tumor cells. Nevertheless, the role of circ_0000527 in retinoblastoma $(\mathrm{RB})$ and its regulatory mechanisms remain largely unknown.

Methods: Real-time PCR (RT-PCR) was used to detect circ_0000527 and miR-646 expression in RB tissues and cells. The LRP6 expression in RB cells was detected by Western blot. The relationship between circ_0000527 expression and the clinicopathological parameters of RB patients was analyzed. Cell proliferation, apoptosis and metastasis were monitored by cell counting kit-8 (CCK-8), flow cytometry, and Transwell assay. The dual-luciferase reporter gene assay and RIP assay were employed to verify the targeting relationship between circ_0000527 and miR-646, miR-646 and LRP6.

Results: Circ_0000527 was highly expressed in both RB and RB cell lines, whose high expression level and degree of differentiation were significantly correlated with the increase in CTNM staging level. Overexpression of circ_0000527 contributed to RB cell proliferation, migration, invasion and suppressed cell apoptosis, while knocking down circ_0000527 inhibited the above malignant biological behavior. The underlying mechanism suggested that functioning as a endogenous competitive RNA, circ_0000527 directly targeted miR-646 and positively regulated LRP6 expression.
\end{abstract}

Conclusion: Circ_0000527 enhances the proliferation and metastasis of RB cells by modulating the miR-646/LRP6 axis.

Keywords: RB, Circ_0000527, miR-646, LRP6, ceRNA

\section{Background}

Retinoblastoma (RB), the most common intraocular malignancy, usually occurs in childhood [1], which accounts for $2-4 \%$ of all malignancies in children under

\footnotetext{
*Correspondence: zhlhz912474715@163.com

${ }^{2}$ Department of Dermatology, The Affiliated Hospital of Qingdao University, No 16, Jiangsu Road, South District, Qingdao 266003, Shandong, China

Full list of author information is available at the end of the article
}

5 years of age and can easily cause vision loss or eye loss or even death [2].

Unlike other linear RNAs, circular RNAs (circRNAs) lack the $3^{\prime}$ and $5^{\prime}$ ends, which protects it from the degradation by RNA exonuclease, so they can be more stably present in tissues and cells $[3,4]$. Accumulating experiments indicate that circRNAs can function as biomarkers for cancer diagnosis and potential therapeutic targets [5]. For instance, circ_0000745 enhances cervical cancer (CC) cell proliferation, migration, invasion and epithelial-mesenchymal transition (EMT) [6]; circ_0058124

(C) The Author(s) 2020. This article is licensed under a Creative Commons Attribution 4.0 International License, which permits use, sharing, adaptation, distribution and reproduction in any medium or format, as long as you give appropriate credit to the original author(s) and the source, provide a link to the Creative Commons licence, and indicate if changes were made. The images or other third party material in this article are included in the article's Creative Commons licence, unless indicated otherwise in a credit line to the material. If material is not included in the article's Creative Commons licence and your intended use is not permitted by statutory regulation or exceeds the permitted use, you will need to obtain permission directly from the copyright holder. To view a copy of this licence, visit http://creativeco mmons.org/licenses/by/4.0/. The Creative Commons Public Domain Dedication waiver (http://creativecommons.org/publicdomain/ zero/1.0/) applies to the data made available in this article, unless otherwise stated in a credit line to the data. 
facilitates the progression of papillary thyroid carcinoma by modulating the NOTCH3/GATAD2A axis [7]; circSERPINE2 up-regulates the expression of YWHAZ and promotes the progression of gastric cancer [8]. However, the researches on expression function and mechanism of circRNA in RB are relatively rare.

MicroRNAs (miRNAs), small non-coding RNAs (ncRNAs) embraceing 19-25 nucleotides, inhibit gene expression by binding to the $3^{\prime}$ untranslated region ( $3^{\prime}-$ UTR) of the target mRNA [9]. Increasing experiments find that miRNAs participate in many aspects of cancer biology, such as cell proliferation, differentiation, apoptosis and metastasis [10]. For instance, in renal clear cell carcinoma, miR-205-5p suppresses VEGFA expression and the PI3K/Akt/mTOR signaling pathway to function as a tumor suppressor [11]; miR-204-5p represses the proliferation and invasion of esophageal squamous cell carcinoma cells by suppressing IL-11 [12]. MiR-646 is considered as a tumor suppressor in several cancers. For example, miR-646 inhibits gastric cancer cell proliferation and EMT-induced metastasis by targeting and suppressing FOXK1 expression [13]. Nonetheless, the expression and function of miR-646 in RB remains largely undefined.

The low-density lipoprotein receptor-related protein 6 (LRP6) is a member of the low-density lipoprotein (LDL) receptor gene family [14]. The protein encoded by this gene acts as a receptor for Wnt or as a co-receptor with Frizzled, involved in the classical Wnt $/ \beta$-catenin signaling pathway [14]. By cascading with the Wnt/ $\beta$-catenin signaling, this gene plays a prominent role in regulating cell differentiation, proliferation, migration, as well as the deterioration of diverse cancer types, including RB [15, 16]. Furthermore, it has been reported that the expression level of LRP6 is modulated by a plurality of miRNAs, such as miR-202-3p and miR-126-3p [17, 18]. However, the mechanism which is responsible for the dysregulation of LRP6 expression in RB has not been fully elucidated.

Interestingly, with bioinformatics analysis, we noticed that LRP6 was a potential target gene of miR-646. Meanwhile, miR-646 was a potential target of circ_0000527. In this study, we investigated the expression pattern of circ_0000527 expression in RB. Furthermore, we explored whether circ_0000527 was involved in the progression of RB via regulating the miR-646 and LRP6.

\section{Materials and methods}

\section{Tissues collection}

45 cases of RB patients and their adjacent tissues were harvested in the Department of Pathology, Affiliated Hospital of Qingdao University. The clinical and pathological features of each patient were collected postoperatively. The study equipped with written informed consent from the guardians of the patients and this study was endorsed by the requirements of the Ethics Committee of the Affiliated Hospital of Qingdao University.

\section{Cell culture and transfection}

The RB cell lines (WERI-RB-1, SO-RB50, Y79, and RB355 cells), human retinal pigment epithelial cells (ARPE-19) and kidney epithelial cell HEK293T cells were purchased from the American Type Culture Center (ATCC, Rockville, MD, USA). All cells were cultured in Dulbecco's Modified Eagle's Medium (DMEM, Thermo Fisher Scientific, Shanghai, China) containing $10 \%$ fetal bovine serum (FBS; Gibco, Grand Island, NY, USA) and 1\% penicillin/ streptomycin (Invitrogen, Carlsbad, CA, USA), and the cells were maintained in a $5 \% \mathrm{CO}_{2}$ humidified incubator at $37^{\circ} \mathrm{C}$. The cells were passaged at intervals of 2 to 3 days, and the cells in the logarithmic growth phase were selected for experiments.

After being trypsinized with trypsin, the cells were then seeded in 24-well plates and cultured in an incubator to continue the culture for $24 \mathrm{~h}$. The circular transcript expression vector possesses two elements termed as the front circular and the back circular frame which were specially designed containing inverted repeat sequences flank. The full-length cDNA of circ_0000527 was amplified in RB cells, and was cloned into the specific vector between two frames. Small interfering RNA (siRNA) against circ_0000527 (si-circ_0000527), the pcDNA 3.1 and the si-NC were purchased from Genechem (Shanghai, China). miR-646 mimics, miR-646 inhibitor, negative control miR-con were purchased from Ribobio (Guangzhou, China), and then they were transfected into WERIRB-1 cells and Y79 cells according to Lipofectamine ${ }^{\mathrm{TM}}$ 3000 (Invitrogen, Carlsbad, CA, USA) transfection reagent instructions. The transfection efficiency was detected $48 \mathrm{~h}$ later.

\section{Quantitative real-time polymerase chain reaction (qRT-PCR)}

According to the manufacturer's instructions, the total RNA was extracted from tissues and cells using TRIzol reagent (Thermo Scientific Hyclone, Utah, USA). After detecting the purity of total RNA with a spectrophotometer, $3 \mu \mathrm{g}$ of total RNA was used as a template, and the RNA was reversely transcribed into cDNA employing the reverse transcription kit (Thermo Scientific Hyclone, Utah, USA). qRT-PCR detection was subsequently performed using BestarTM qPCR MasterMix. GAPDH and U6 were considered as internal parameters, and the $2^{-\Delta \Delta C T}$ method was employed to calculate the relative expression levels of the molecules. The primer sequences were available from Thermo Fisher Scientific (Shanghai, China), and more details could be found in Table 1 . 


\section{The cell counting kit (CCK-8) assay}

CCK-8 was adopted to examine the effects of circ_0000527 and si-circ_0000527 or miR-646 mimics and miR-646 inhibitors on RB cell viability. RB cells were seeded into 96-well plates at a density of $1 \times 10^{3}$ cells per well. The cells were cultured at $37^{\circ} \mathrm{C}$ for 24,48 , and $72 \mathrm{~h}$, and incubated with CCK-8 solution $(10 \mu \mathrm{L})$ (Dojindo, Kumamoto, Japan) for $10 \mathrm{~min}$, and then the absorbance in each well was measured at $450 \mathrm{~nm}$ using a microplate reader (Bio-Rad, Hercules, CA, USA).

\section{Flow cytometry}

Y79 and WERI-RB-1 cells in the logarithmic growth phase were collected, and single-cell suspension was prepared and fixed in $70 \%$ ethanol at $4{ }^{\circ} \mathrm{C}$ overnight. Afterwards, the cells were resuspended in binding buffer and the concentration was modulated to $1 \times 10^{4} / \mathrm{ml}$. The cells were stained according to the proportion of $10 \mu \mathrm{L}$ Annexin V-fluorescein isothiocyanate (Annexin V-FITC) and $5 \mu \mathrm{L}$ propidium iodide (PI) kit (BD Biosciences, San Diego, CA, USA) per $1 \mathrm{ml}$ cell suspension and incubated in dark at room temperature for $15 \mathrm{~min}$, followed by the addiditon of $400 \mu \mathrm{L}$ binding buffer. After that, flow cytometry (BD Biosciences, San Jose, CA,USA) was performed within $1 \mathrm{~h}$ to analyze the cell apoptosis.

\section{Transwell migration and invasion assays}

Migration and invasion were assessed using a Transwell chamber (Costar, Cambridge, MA, USA) without Matrigel (for migration analysis) and with Matrigel (for invasion assay), respectively. RB cell suspension $\left(2 \times 10^{4}\right.$ cells in $200 \mu \mathrm{L}$ serum-free medium) was added to the upper compartment and the medium supplemented with $20 \%$ FBS $(500 \mu \mathrm{L})$ was added to the lower compartment. After cells were cultured at $37{ }^{\circ} \mathrm{C}$ for $24 \mathrm{~h}$, the cells passing through the membrane were fixed with $4 \%$ paraformaldehyde, stained with crystal violet, and counted.

Table 1 Primer sequences used for qRT-PCR

\begin{tabular}{|c|c|}
\hline \multirow[t]{2}{*}{ circ_0000527 } & F:TCAACCAGGTGGATGTGTGG \\
\hline & R: GACTCGCTCGAGAGGGGTTA \\
\hline \multirow[t]{2}{*}{ miR-646 } & F: ACACTCCAGCTGGGAAGCAGCTGCCTC \\
\hline & R: CTCAACTGTGCTGCATTAGTTAGCTCAGA \\
\hline \multirow[t]{2}{*}{ U6 } & F: CGCTTCGGCAGCACATATACTA \\
\hline & R: CGCTTCACGAATTTGCGTGTCA \\
\hline \multirow[t]{2}{*}{ LRP6 } & F: AGG CACTTACTTCCCTGCAA \\
\hline & R: GGGCACAGGTTCTGAATCAT \\
\hline \multirow[t]{2}{*}{ GAPDH } & F: CGGATTTGGTCGTATTGGG \\
\hline & R: CTGGAAGATGGTGATGGGATT \\
\hline
\end{tabular}

$F$ forward, $R$ reverse, $R T$ reverse transcription

\section{Western blot}

The total protein of each group was extracted using RIPA lysis buffer (Beijing solarbio science \& technology co., Ltd, Beijing, China) according to the manufacturer's instructions. Equal amounts of protein from each group of cells were subjected to SDS-PAGE electrophoresis and then transferred to a polyvinylidene fluoride (PVDF) membrane. After being blocked for $1 \mathrm{~h}$ at room temperature in $5 \%$ fat-free milk, the membrane was incubated with specific primary antibody at $4{ }^{\circ} \mathrm{C}$ overnight. The primary antibody was obtained from Abcam (Shanghai, China): Anti-LRP6 (Abeam, ab134146, 1:500). Then the membrane was incubated with an horse radishperoxidase (HRP)-labeled secondary antibody (Abcam, ab216773, 1:5000) at room temperature for $1 \mathrm{~h}$. After the procedure of wash, the membrane was exposed with ECL chromogenic reagent (Millipore, Bedford, MA, USA), and then was exposed to film to observe the bands.

\section{RNA immunoprecipitation (RIP) assay}

The EZMagna RIP kit (Merck, Darmstadt, Germany) was used for the RIP experiment in accordance with the manufacturer's instructions. Anti-Argonaute 2 (AGO2) or anti-IgG antibody was coupled to magnetic beads. The cells were transfected with miR-646 or control miRNA, before RIP lysis buffer was utilized to lyse the cells, after which the lysate was incubated with magnetic beads for $6 \mathrm{~h}$ at $4{ }^{\circ} \mathrm{C}$. In order to remove the protein, the magnetic beads were rinsed and incubated with the protease $\mathrm{K}$ buffer. Next, RNA was immunoprepcipitated, and reverse transcription was performed with Prime-Script RT Master Mix (TaKaRa, Dalian, China). Finally, the expression of circ_0000527 was analyzed by qRT-PCR.

\section{Dual luciferase reporter assay}

TargetScan (http://www.targetscan.org) was used to predict potential binding sites. Dual luciferase reporter assay was employed to detect the binding relationship between circ_0000527 and miR-646, miR-646 and the 3'UTR of LRP6. HEK293T cells were cultured in 12-well plates and co-transfected with vectors containing wild-type or mutant circ_0000527/3'UTR of LRP6 and miR-646 mimics or control miRNA. Firefly and Renilla luciferase activities were measured $48 \mathrm{~h}$ after the transfection employing the dual luciferase reporter system (Promega, Madison, WI, USA). Luciferase activity was calculated as the ratio of firefly luciferase intensity to Renilla luciferase intensity.

\section{Statistical analysis}

Data analysis was performed using SPSS 22.0. All experimental data were expressed as mean $\pm S D$, and differences between the two groups were compared using the 
$t$-test. In this study, $P<0.05$ was considered as statistically significant.

\section{Result}

High expression of circ_0000527 in RB tissues and cells and its clinical significance

To explore the expression characteristics of circ_0000527 in RB tissues and cells, qRT-PCR was performed. The results authenticated that circ_0000527 expression in RB tissues was significantly higher than that in adjacent tissues (Fig. 1a). In addition, compared with retinal pigment epithelial cells ARPE-19 cells, circ_0000527 expression in RB cells (WERI-RB-1, SO-RB50, Y79 and RB355 cell lines) was significantly increased (Fig. 1b). Likewise, by analyzing the relationship between the expression level of circ_0000527 and the clinicopathological parameters of RB patients, we found that the high expression level of circ_0000527 was significantly correlated with the degree of differentiation and the increase of cTNM staging level (Table 2). These studies suggested that circ_0000527 played an oncogenic role in RB.

\section{circ_0000527 enhanced RB cell proliferation, migration} and invasion and suppressed the apoptosis

To further probe the role of circ_0000527 in RB, we transfected circ_0000527 plasmid and siRNA targeting circ_0000527 into Y79 and WERI-RB-1 cells, respectively. The transfection was confirmed by qRT-PCR (Fig. 1c, d). After the cell models were successfully constructed, cell proliferation and apoptosis were detected by CCK-8 and flow cytometry, respectively, the data of which manifested that overexpression of circ_0000527 notably enhanced Y79 cell proliferation and suppressed apoptosis compared to the control group (Fig. 2a, b). Transwell assay confirmed that overexpression of circ_0000527 promoted cell migration (Fig. 2c) and invasion (Fig. 2d)
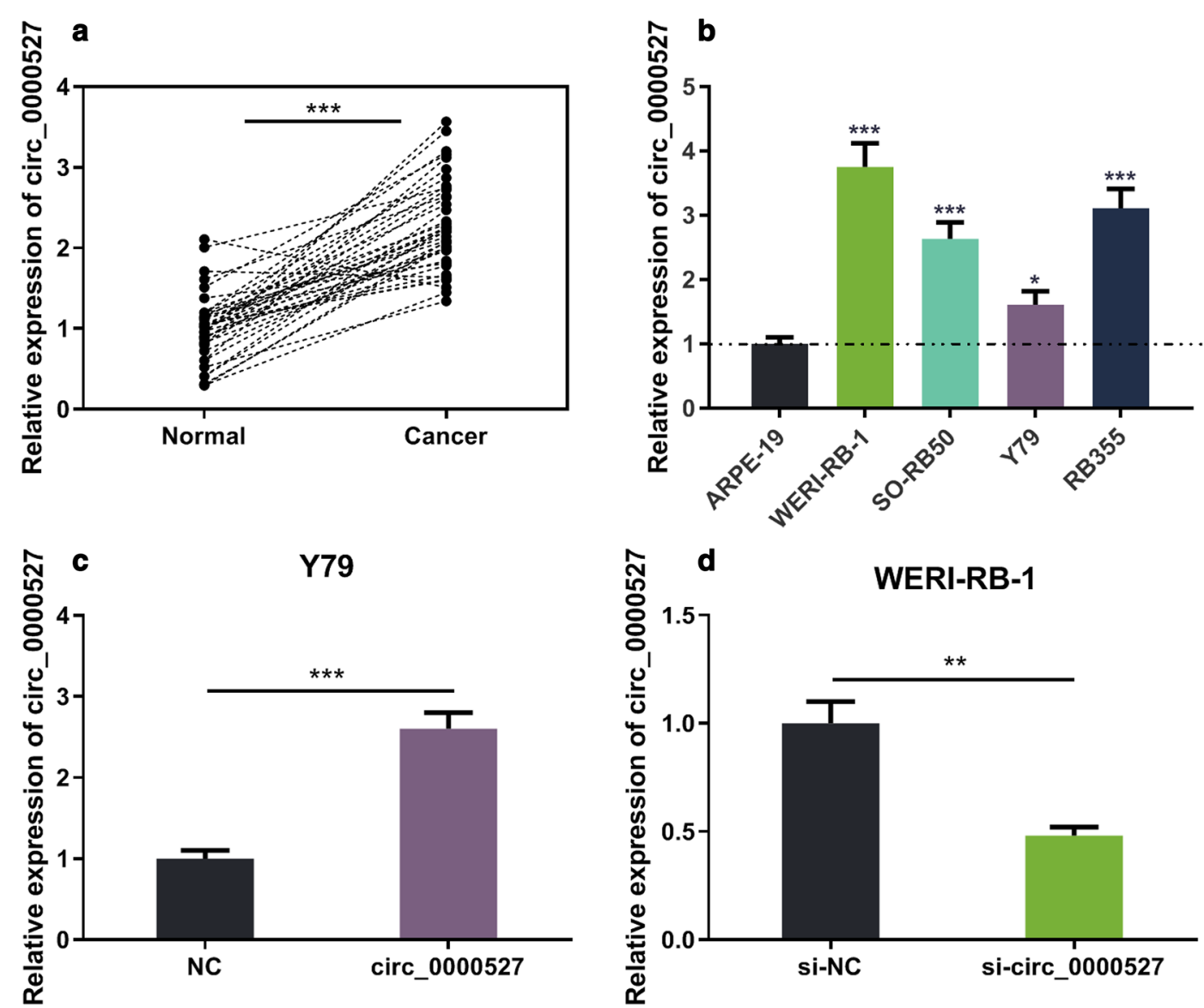

Fig. 1 circ_0000527 expression was detected in RB tissues and cell lines. a The expression of cric_0000527 in 45 cases of paired RB and paracancerous tissues was detected by qRT-PCR. b The expression of cric_0000527 in retinal pigment epithelial cells (ARPE-19) and RB cells was detected by qRT-PCR. c The expression level of cric_0000527 was detected by qRT-PCR after transfecting the overexpressing cric_0000527 plasmid into Y79 cells. d After transfecting cric_0000527 targeting siRNA into WERI-RB-1 cells, the expression level of cric_0000527 was detected by qRT-PCR. ${ }^{*} P<0.05$, ${ }^{* *} P<0.01$, and ${ }^{* *} P<0.001$ 
Table 2 Correlations between circ_0000527 and clinical characteristics in RB patients

\begin{tabular}{|c|c|c|c|c|}
\hline \multirow[t]{2}{*}{ Pathological indicators } & \multirow[t]{2}{*}{$\begin{array}{l}\text { Number } \\
\text { of patients }\end{array}$} & \multicolumn{2}{|c|}{$\begin{array}{l}\text { circ_0000527 } \\
\text { expression }\end{array}$} & \multirow[t]{2}{*}{$P$ value } \\
\hline & & High & Low & \\
\hline All cases & 25 & 14 & 11 & \\
\hline \multicolumn{5}{|l|}{ Age (years) } \\
\hline$\geq 5$ & 4 & 1 & 3 & 0.173 \\
\hline$<5$ & 21 & 13 & 8 & \\
\hline \multicolumn{5}{|l|}{ Gender } \\
\hline Male & 12 & 6 & 6 & 0.561 \\
\hline Female & 13 & 8 & 5 & \\
\hline \multicolumn{5}{|c|}{ Tumor enucleated location } \\
\hline Right & 13 & 8 & 5 & 0.561 \\
\hline Left & 12 & 6 & 6 & \\
\hline \multicolumn{5}{|l|}{ Differentiation } \\
\hline Well and moderate & 15 & 6 & 9 & $0.048^{*}$ \\
\hline Poor & 10 & 8 & 2 & \\
\hline \multicolumn{5}{|l|}{ T classification } \\
\hline $\mathrm{T} 1+\mathrm{T} 2$ & 15 & 7 & 8 & 0.250 \\
\hline $\mathrm{T} 3+\mathrm{T} 4$ & 10 & 7 & 3 & \\
\hline \multicolumn{5}{|l|}{ N classification } \\
\hline NO & 11 & 4 & 7 & 0.080 \\
\hline $\mathrm{N} 1+\mathrm{N} 2$ & 14 & 10 & 4 & \\
\hline \multicolumn{5}{|l|}{ cTNM stage } \\
\hline $1+\|$ & 6 & 1 & 5 & $0.026^{*}$ \\
\hline$I I I+I V$ & 19 & 13 & 6 & \\
\hline \multicolumn{5}{|l|}{ Largest tumor base (mm) } \\
\hline$\geq 15$ & 9 & 7 & 2 & 0.100 \\
\hline$<15$ & 16 & 7 & 9 & \\
\hline
\end{tabular}

compared to the control group, while knocking down circ_0000527 inhibited cell proliferation, migration and invasion, and enhanced cell apoptosis (Fig. 2a-d). These results suggested that circ_0000527 accelerated RB cell progression.

Targeting relationship between circ_0000527 and miR-646 Through the StarBase database 3.0 (http://starbase. sysu.edu.cn/panCancer.php), we found that a potential binding site existed between circ_0000527 and miR-646 (Fig. 3a). To verify whether circ_0000527 absorbed miR-646, dual luciferase reporter assay was carried out. The results implied that the miR-646 mimics markedly reduced the luciferase activity of the wildtype circ_0000527 reporter compared to the control group, whereas the luciferase activity of the mutant circ_0000527 reporter was not significantly altered by miR-646 mimics (Fig. 3b). Furthermore, RIP assay confirmed that circ_0000527 and miR-646 were enriched in
Ago2-containing microribonucleoproteins compared to control IgG (Fig. 3c). Based on the Pearson correlation analysis, we found a negative correlation between the expression of cric_0000527 and miR-646 in RB samples (Fig. 3d). Additionally, we found that the upregulation of circ_0000527 significantly suppressed the expression of miR-646, and knockdown of circ_0000527 enhanced the expression of miR-646 in RB cell lines (Fig. 3e). Moreover, there was no significant change in the expression of circ_0000527 after up-regulation or inhibition of miR646 (Fig. 3f). In summary, circ_0000527 was an upstream molecule that could adsorb miR-646 and negatively regulate its expression.

\section{miR-646 inhibited the malignant phenotypes of RB cells}

The data mentioned above suggested that miR-646 acted as a downstream molecule of circ_0000527. To investigate the expression and role of miR-646 in RB, qRT-PCR was employed to detect the expression of miR-646 in RB tissues and cells. The results revealed that miR-646 expression was remarkably decreased in RB cancer tissues compared with paracancerous tissues (Fig. 4a); consistently, miR-646 expression was notably decreased in RB cells compared with ARPE-19 cells (Fig. 4b). Then, the miR-646 mimics and the miR-646 inhibitor were transfected into WERI-RB-1 cells and Y79 cells, respectively. The model for high expression and low expression of miR-646 was successfully constructed and verified by qRT-PCR (Fig. 4c, d). CCK-8, flow cytometry, and transwell assay confirmed that overexpression of miR-646 inhibited cell proliferation, migration, and invasion, and enhanced cell apoptosis. On the contrast, inhibition of miR-646 enhanced cell proliferation, migration and invasion, and repressed cell apoptosis (Fig. 4e-h). These results revealed that miR-646 exerted a tumor-suppressive effect on RB.

circ_0000527/miR-646 axis modulated RB cell proliferation, apoptosis, migration and invasion

In order to explore the function of the circ_0000527/ miR-646 axis in regulating the biological behaviors of RB cells, miR-646 mimics and circ_0000527 overexpressing plasimds were co-transfected into Y79 cells, and the miR-646 inhibitor and circ_0000527 siRNA were co-transfected into WERI-RB-1 cells. The transfection was validated by qRT-PCR (Fig. 5a). It was confirmed that co-transfection of miR-646 mimics reversed the effects of circ_0000527 overexpression on RB cells; similarly, co-transfection of miR646 inhibitors reversed the effects of circ_0000527 knockdown (Fig. 5b-f). These studies indicated that the circ_0000527/miR-646 axis was engaged in RB progression. 


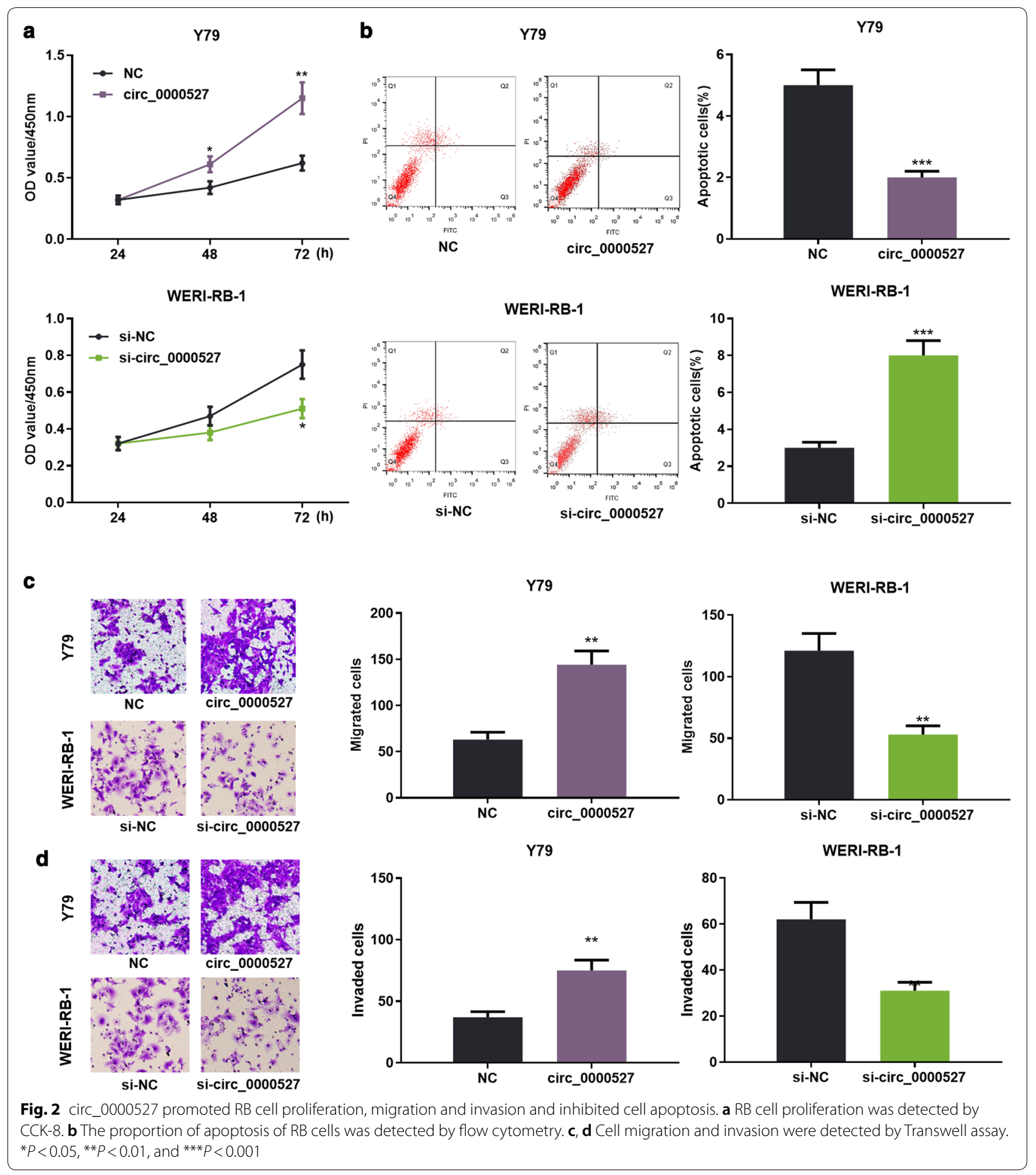

circ_0000527/miR-646 axis regulated the expression of LRP6

To further explore the downstream molecules of miR646, we found through the TargetScan database that there was a potential base-complementary binding site between
miR-646 and the 3'UTR of LRP6 (Fig. 6a). To verify whether miR-646 binded to LRP6, dual luciferase reporter assay was carried out and the results showed that the miR646 mimics remarkably decreased the luciferase activity of the wild-type LRP6 3'UTR -WT reporter compared to 

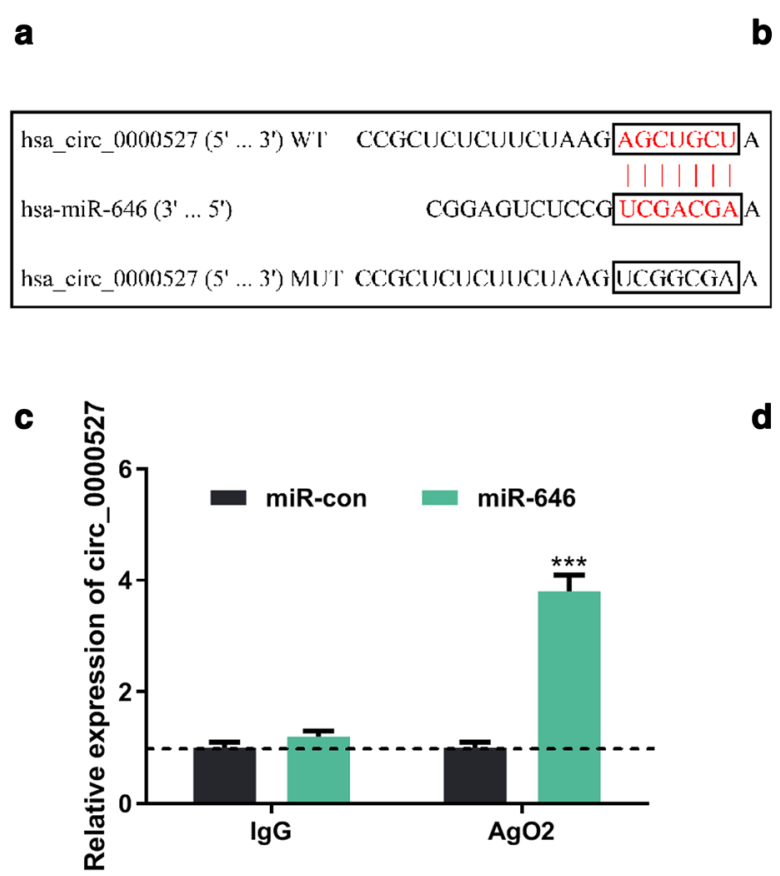

d
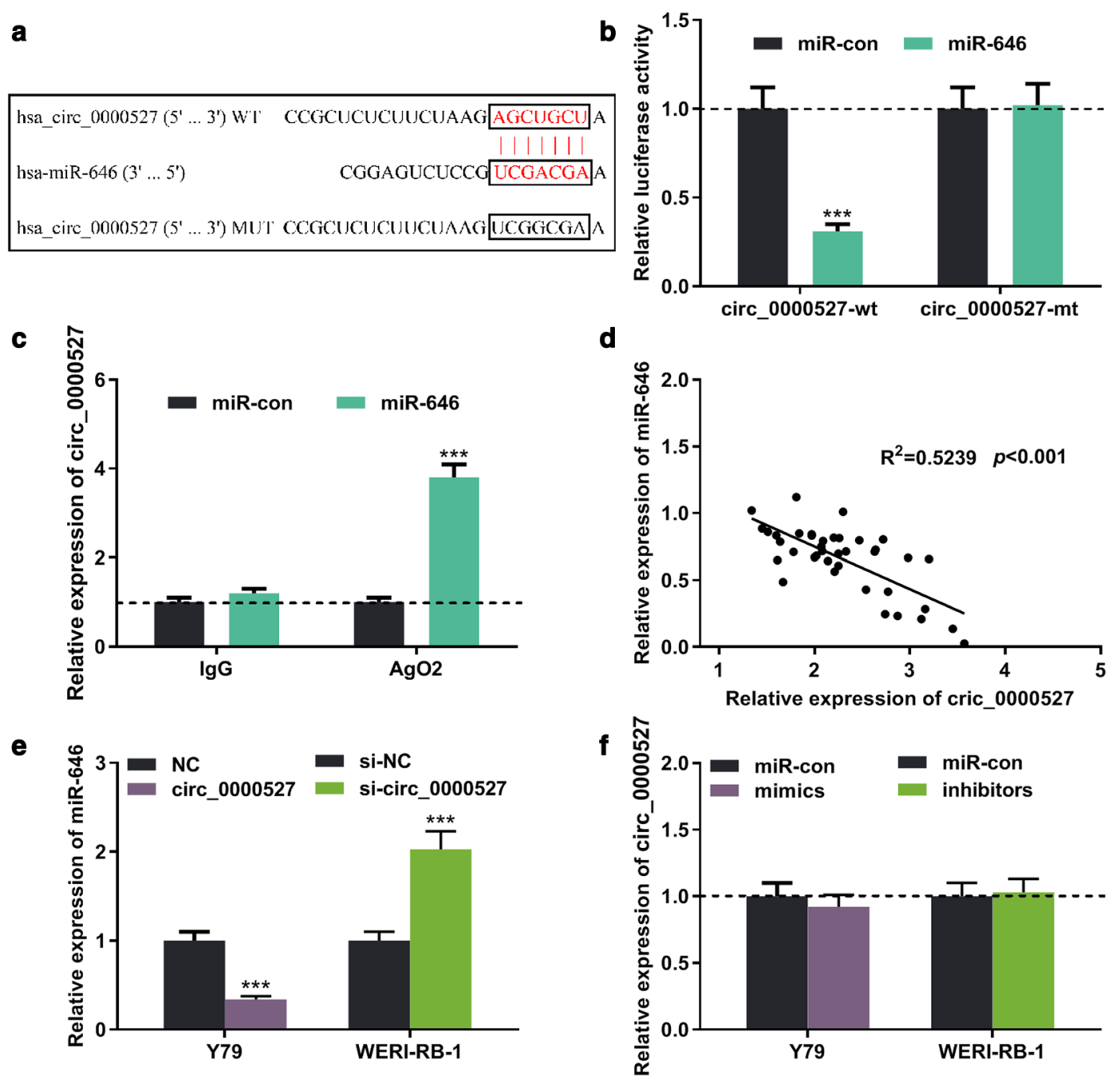

Fig. 3 circ_0000527 adsorbed miR-646. a The binding site between cric_0000527 and miR-646. b The binding relationship between cric_0000527 and miR-646 was verified by luciferase activity assay in 293T cells. c The interaction between cric_0000527 and miR-646 was detected by the RIP assay in 293T cells. d The correlation between cric_0000527 and miR-646 expression in RB samples was analyzed by Pearson analysis. e The expression of miR-646 was detected by qRT-PCR after up-regulating or down-regulating cric_0000527 in RB cells. f The expression of cric_0000527 was detected by qRT-PCR after up-regulating or down-regulating miR-646. ${ }^{* *} P<0.001$

the control, but did not change that of the mutant reporter (Fig. 6b). In addition, qRT-PCR and Western blot assay verified that the expression of LRP6 mRNA and the protein was increased by circ_0000527 overexpression or miR-646 inhibition in RB cells, while knocking down circ_0000527 or upregulating miR-646 caused a decrease in LRP6 expression (Fig. 6c, d). Besides, the expression of circ_0000527 was verified to be positively correlated with that of LRP6 in $\mathrm{RB}$ and paracancerous tissues (Additional file 1: Figure S1). These results implied that LRP6 was negatively regulated by miR-646 and was positively regulated by circ_0000527.

\section{Discussion}

Recently, ncRNAs including circRNA have increasingly become a hot topic in the field of cancer research [19, 20]. In-depth study of the role of circRNA in RB and its regulatory mechanisms is of substantial value to provide a theoretical basis for elucidating the mechanism of $\mathrm{RB}$ progression [21]. In this study, we found for the first time that circ_0000527 expression was remarkably up-regulated in RB tissues and cell lines. Furthermore, its high expression level is significantly associated with adverse clinical features of RB patients. In terms of mechanism, circ_0000527 accelerated the progression of RB by adsorbing miR-646 and activating LRP6 expression. 

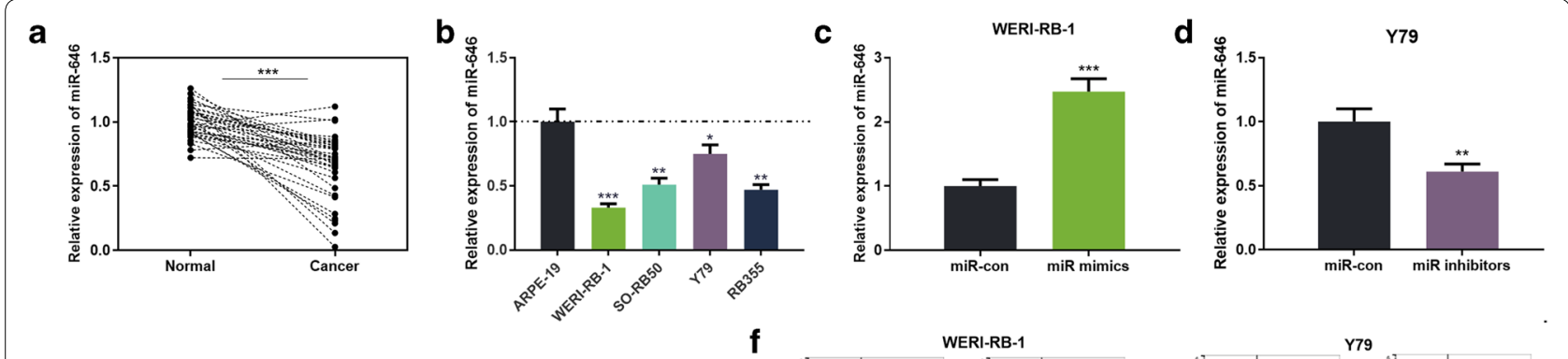

$\mathbf{e}$
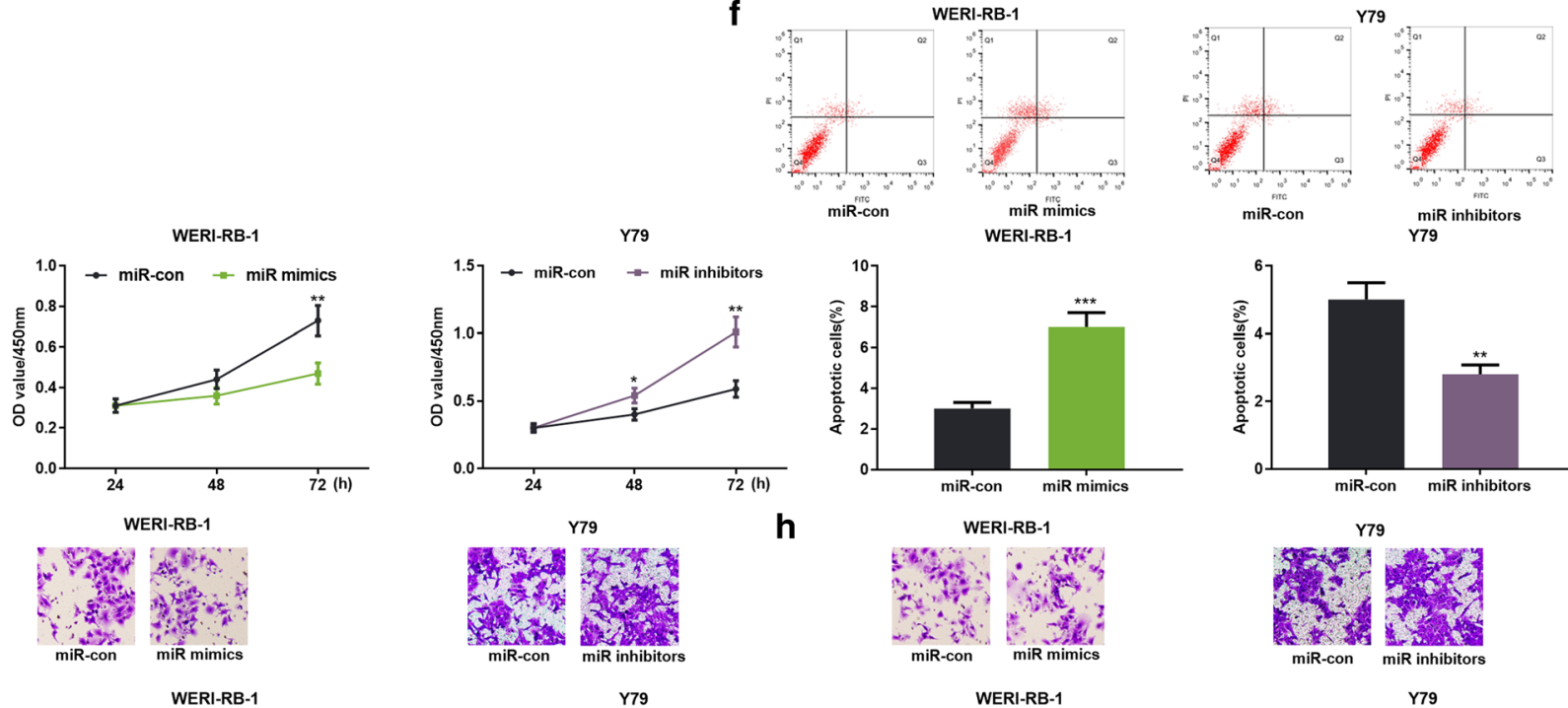

h
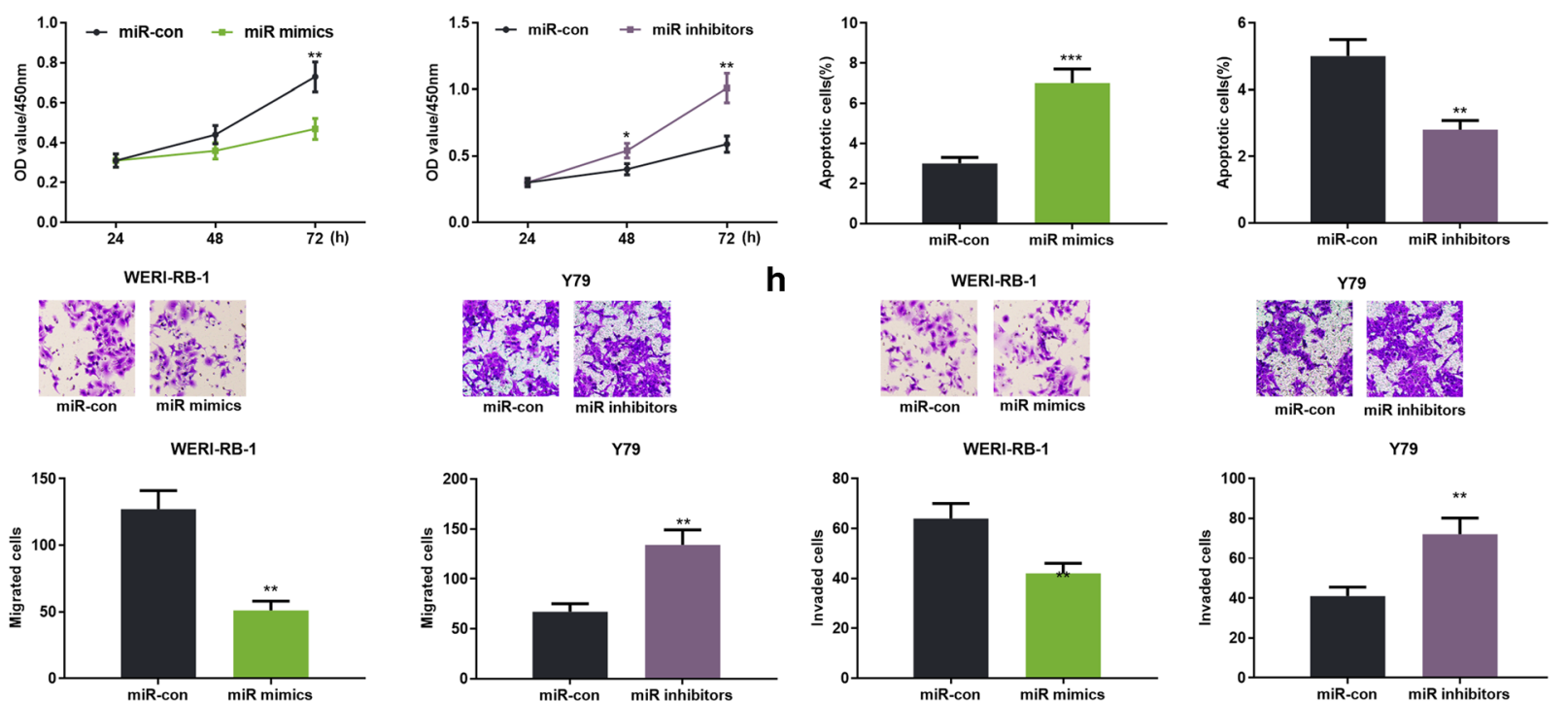

Fig. 4 miR-646 functioned as a tumor suppressor in RB. a The expression of miR-646 in 45 cases of paired RB and paracancerous tissues was detected by qRT-PCR. b The expression of miR-646 in retinal pigment epithelial cells (ARPE-19) and RB cells was detected by qRT-PCR. $\mathbf{c}$, $\mathbf{d}$ The expression level of miR-646 was detected by qRT-PCR after RB cells were transfected with miR-646 mimics and inhibitors. e RB proliferation was detected by the CCK-8. f Flow cytometry was used to detect apoptosis of RB cells. $\mathbf{g}, \mathbf{h}$ Transwell assay was used to detect RB cell migration and invasion. ${ }^{* *} P<0.001$, and ${ }^{* *} P<0.01$

CircRNAs, derived from reverse splicing or exon skipping of pre-mRNAs [5], is stable due to its special ring structure and resistant to exonuclease [22]. The role of circRNA in tumorigenesis and cancer progression has attracted widespread attention. Functionally, circRNA participates in the formation of tumors, regulating malignant biological behaviors of cancer cells such as proliferation, apoptosis and metastasis. It is reported that circ_0001649 suppresses RB cell proliferation and apoptosis by inhibiting AKT/mTOR signaling pathway, and its low expression level indicates poor prognosis in patients [23]; circ_ODC1 positively regulates SKP2 and promotes RB cell proliferation [24]. In this study, we found for the first time that the expression level of circ_0000527 in RB tissues and cells was significantly increased, and its high expression was significantly correlated with the lower the degree of differentiation and increased cTNM staging. These results indicated that circ_0000527 was expected to be a predictor of poor prognosis of RB patients. In addition, the proliferation, migration and invasion of the cells were remarkably promoted by circ_0000527 overexpression, while the proportion of apoptosis of cells was remarkably reduced. Conversely, knocking down circ_0000527 exerted the opposite effect. The above results suggested that circ_0000527 was involved in promoting the progression of $\mathrm{RB}$ and demonstrated that it had an important role in RB. 

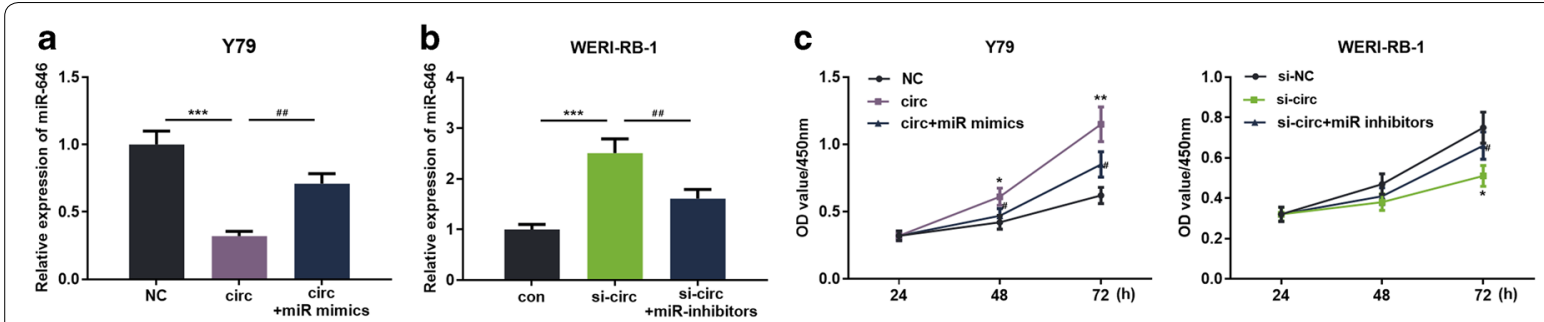

d
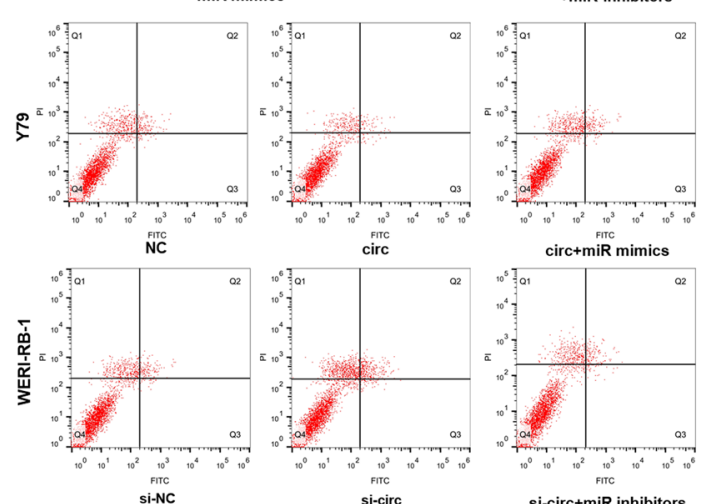

circ
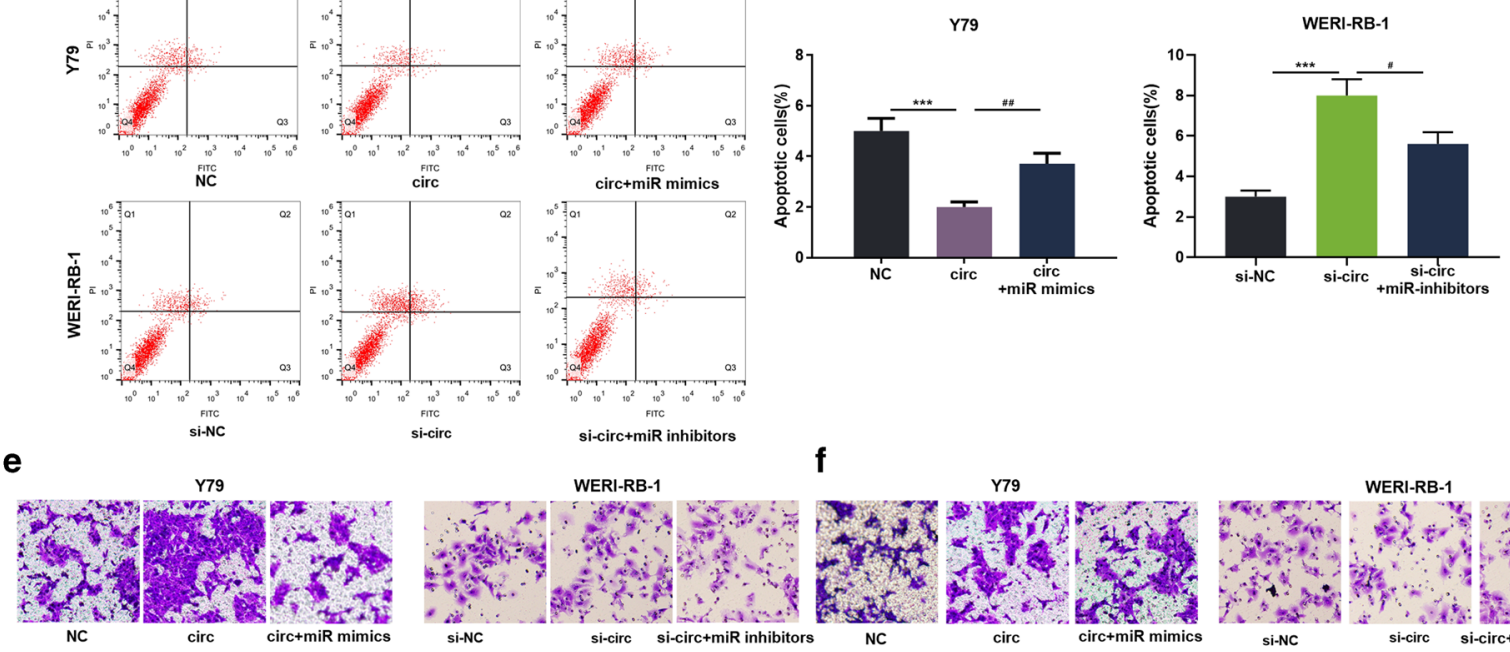

si-circ+miR inhibitors

WERI-RB-1

f
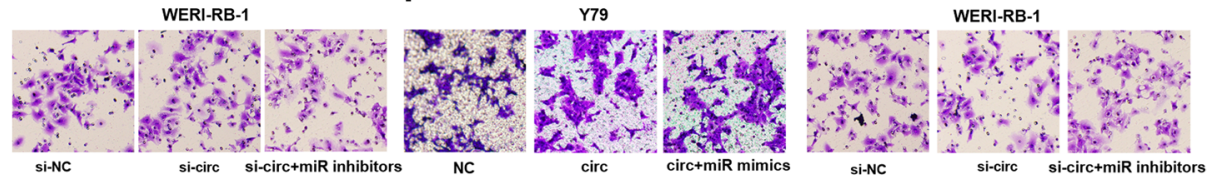

Y79

WERI-RB-1
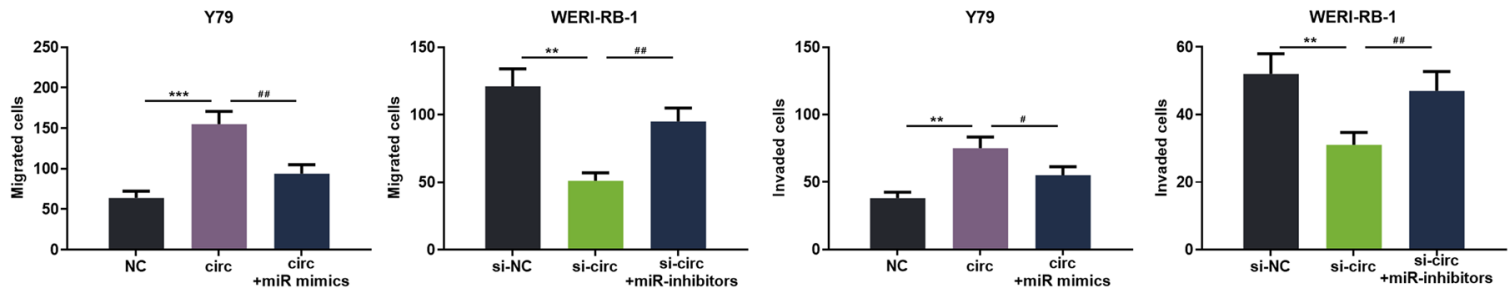

Fig. 5 circ_0000527/miR-646 axis regulated RB cell proliferation, apoptosis, migration and invasion. a, b Transfection efficiency was detected by qRT-PCR. c The proliferation was detected by the CCK-8 method. $\mathbf{d}$ Flow cytometry was used to detect apoptosis of RB cells. e, $\mathbf{f}$ Transwell assay was used to detect RB cell migration and invasion. ${ }^{*} P<0.05,{ }^{* *} P<0.01$, ${ }^{* * *} P<0.001$, NC vs. circ_0000527 or si-NC vs. si-NC. ${ }^{\#} P<0.05,{ }^{\# \#} P<0.01$, circ_0000527 vs. circ_0000527 + miR-646 or si-circ_0000527 vs. si-circ_0000527 + miR-646 inhibitors

MiRNAs are also reported to be engaged in the regulation of the tumorigenesis and progression of a variety of human tumors $[25,26]$. MiR-646 is regarded as a tumor suppressor in many human cancers. For instance, in lung cancer, miR-646 suppresses cell proliferation and metastasis by negatively regulating the EGFR/Akt pathway [27]; in colorectal cancer, miR-646 represses NOB1 to inhibit cancer progression [28]. MiR-646 suppresses clear cell renal carcinoma cell metastasis by downregulating nin one binding protein [29]. MiR-646 is involved in inhibiting proliferation and metastasis of non-small cell lung cancer by binding to FGF2 and CCND2 [30]. These studies suggested that miR-646 plays an anticancer role in a variety of tumors. In this study, we found a significant decrease in miR-646 expression in RB tissues and cell lines. In vitro assays confirmed that miR-646 overexpression remarkably repressed RB cell proliferation and metastasis and enhanced cell apoptosis, whereas the inhibition of miR-646 significantly promoted the aforementioned malignant biological behaviors. It should be noted that previous researches show that circRNAs regulate gene expression by adsorbing miRNAs [31]. In view of the distinct roles of circ_0000527 and miR-646 in RB progression, we made a hypothesis that there was such a relationship between them. As we expected, we identified a potential binding site between miR-646 and circ_0000527, and we found that circ_0000527 could negatively regulate miR-646. These data implied that in 

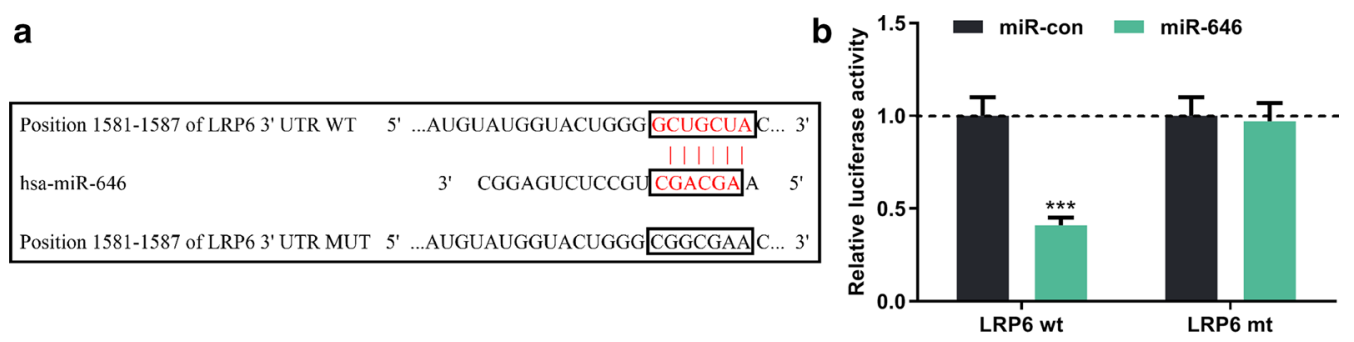

C
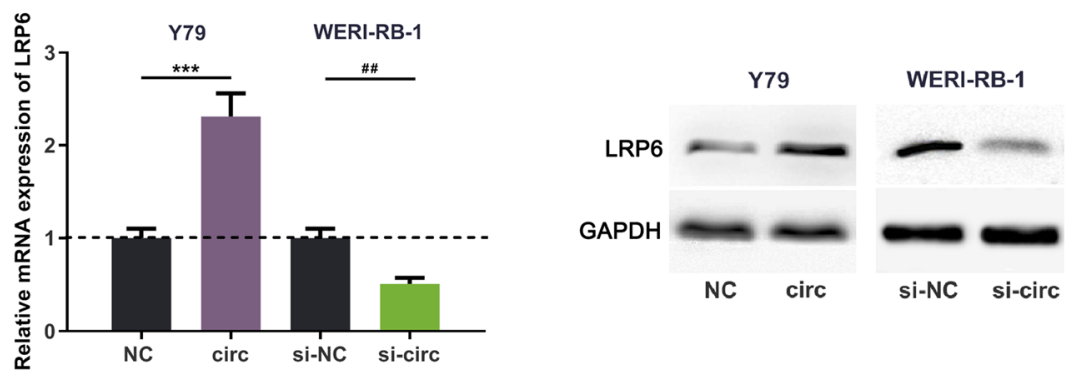

d
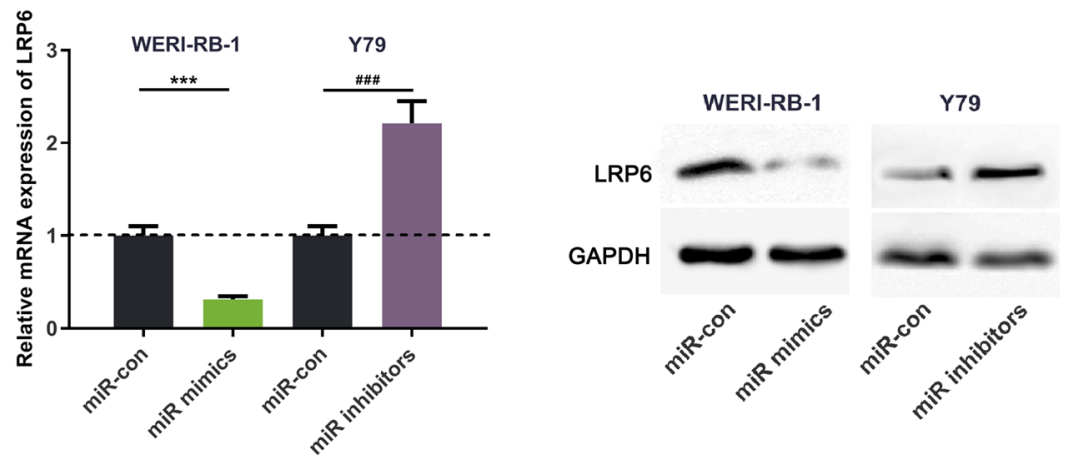

Fig. 6 Regulatory effect of circ_0000527/miR-646 axis on the expression of LRP6. a The binding site between miR-646 and the 3'UTR of LRP6. b The binding relationship between miR-646 and 3'UTR of LRP6 was verified by luciferase activity assay. c The expression of LRP6 was detected by qRT-PCR and Western blot after up-regulation or down-regulation of cric_0000527, respectively. $\mathbf{d}$ The expression of LRP6 was detected by qRT-PCR and Western blot after up-regulation or inhibition of miR-646, respectively. ${ }^{* *} P<0.001$, and ${ }^{* *} P<0.01$

RB, the functions of circ_0000527 were partly mediated by miR-646.

LRP6 is a transmembrane protein involved in receptormediated endocytosis of lipoproteins and protein ligands [32]. This protein forms a complex with transmembrane receptor members of the Frizzled family and acts as a coreceptor in the classical Wnt/ $\beta$-catenin signaling cascade [33]. It is reported that LRP6 is up-regulated in various tumors, including colon cancer, breast cancer, as well as RB [16, 34, 35]. In this study, we found that the upregulation of miR-646 and knockdown of circ_0000527 induced a decrease in LRP6 expression in RB cells. On the other hand, inhibition of miR-646 or overexpression of circ_0000527 enhances LRP6 expression. Therefore, we concluded that the circ_0000527/miR-646 axis contributed to the dysregulation of LRP6 in RB.

\section{Conclusion}

In conclusion, our study presents a novel body of experimental data in support of circ_0000527 as a tumorpromoting circRNA in RB. We find for the first time the high expression level of circ_0000527 in RB tissues and cells, whose high expression is related to RB patients' adverse clinicopathological parameters. In terms of mechanism, we confirm that circ_0000527 promotes cell proliferation, migration, and invasion by modulating the miR-646/LRP6 axis, which is expected to elucidate the mechanism of RB progression. Therefore, circ_0000527/miR-646/LRP6 axis can serve as a promising and effective therapeutic target for $\mathrm{RB}$ patients. However, the sample of this research study is limited to a single center, and and larger sample size is needed in the future to further confirm this conclusion. 


\section{Supplementary information}

Supplementary information accompanies this paper at https://doi. org/10.1186/s12935-020-01396-4.

Additional file 1: Figure S1. The correlations between the expression levels of circ_0000527 and LRP6. (A) The expression level of circ_0000527 was positively correlated with that of LRP6 in RB. (B) The expression level of circ_0000527 was positively correlated with that of LRP6 in paracancerous tissues.

\section{Abbreviations}

circRNAs: Circular RNAs; RB: Retinoblastoma; RT-PCR: Real-time PCR; CCK-8: Cell counting kit-8; miRNAs: MicroRNAs; 3'-UTR: 3' untranslated region; LRP6: Lowdensity lipoprotein receptor-related protein 6; DMEM: Dulbecco's Modified Eagle's Medium; FBS: Fetal bovine serum; siRNA: Small interfering RNA; PVDF: Polyvinylidene fluoride; AGO2: Anti-argonaute 2.

\section{Acknowledgements}

Not applicable.

\section{Authors' contributions}

Conceived and designed the experiments: YLL; Performed the experiments: LZ, JW, YJL, YXJ, LLW, YQC, YLL, LWZ; Statistical analysis: LZ; Wrote the paper: LZ, $Y L L, X Y D$. All authors read and approved the final manuscript.

\section{Funding}

Not applicable.

\section{Availability of data and materials}

The data used to support the findings of this study were available from the corresponding author upon request.

\section{Ethics approval and consent to participate}

Our study was approved by the Ethics Review Board of the Affiliated Hospital of Qingdao University.

\section{Consent for publication}

All authors read the final manuscript and agreed to publish it.

\section{Competing interests}

The authors declare that they have no competing interests.

\section{Author details}

${ }^{1}$ Department of Pathology, The Affiliated Hospital of Qingdao University, Qingdao 266003, Shandong, China. ${ }^{2}$ Department of Dermatology, The Affiliated Hospital of Qingdao University, No 16, Jiangsu Road, South District, Qingdao 266003, Shandong, China.

Received: 5 February 2020 Accepted: 1 July 2020

Published online: 10 July 2020

\section{References}

1. Le Rhun E, Preusser M, Roth P, Reardon DA, van den Bent M, Wen P, Reifenberger G, Weller M. Molecular targeted therapy of glioblastoma. Cancer Treat Rev. 2019:80:101896.

2. Wang S, Du S, Lv Y, Zhang F, Wang W. MicroRNA-665 inhibits the oncogenicity of retinoblastoma by directly targeting high-mobility group box 1 and inactivating the Wnt/beta-catenin pathway. Cancer Manag Res. 2019:11:3111-23.

3. Yu T, Wang Y, Fan Y, Fang N, Wang T, Xu T, Shu Y. CircRNAs in cancer metabolism: a review. J Hematol Oncol. 2019;12:90

4. Bach DH, Lee SK, Sood AK. Circular RNAs in cancer. Mol Ther Nucl Acids. 2019:16:118-29.

5. Wu J, Qi X, Liu L, Hu X, Liu J, Yang J, Yang J, Lu L, Zhang Z, Ma S, Li H, Yun X, Sun T, Wang Y, Wang Z, Liu Z, Zhao W. Emerging epigenetic regulation of circular RNAs in human cancer. Mol Ther Nucl Acids. 2019:16:589-96.
6. Jiao J, Zhang T, Jiao X, Huang T, Zhao L, Ma D, Cui B. Hsa_circ_0000745 promotes cervical cancer by increasing cell proliferation, migration, and invasion. J Cell Physiol. 2020;235(2):1287-95.

7. Yao Y, Chen X, Yang H, Chen W, Qian Y, Yan Z, Liao T, Yao W, Wu W, Yu T, Chen Y, Zhang Y. Hsa_circ_0058124 promotes papillary thyroid cancer tumorigenesis and invasiveness through the NOTCH3/GATAD2A axis. J Exp Clin Cancer Res. 2019:38:318.

8. Liu J, Song S, Lin S, Zhang M, Du Y, Zhang D, Xu W, Wang H. Circ-SERPINE2 promotes the development of gastric carcinoma by sponging miR-375 and modulating YWHAZ. Cell Prolif. 2019;52:e12648.

9. Khan AQ, Ahmed El, Elareer NR, Junejo K, Steinhoff M, Uddin S. Role of miRNA-regulated cancer stem cells in the pathogenesis of human malignancies. Cells. 2019;8(8):840.

10. Ye J, Xu M, Tian X, Cai S, Zeng S. Research advances in the detection of miRNA. J Pharm Anal. 2019;9:217-26.

11. Huang J, Wang $X$, Wen G, Ren Y. miRNA2055p functions as a tumor suppressor by negatively regulating VEGFA and PI3K/Akt/mTOR signaling in renal carcinoma cells. Oncol Rep. 2019;42(5):1677-88.

12. Tang J, Li Z, Zhu Q, Wen W, Wang J, Xu J, Wu W, Zhu Y, Xu H, Chen L. miR-204-5p regulates cell proliferation, invasion, and apoptosis by targeting IL-11 in esophageal squamous cell carcinoma. J Cell Physiol. 2020;235(3):3043-55

13. Zhang P, Tang WM, Zhang H, Li YQ, Peng Y, Wang J, Liu GN, Huang XT, Zhao JJ, Li G, Li AM, Bai Y, Chen Y, Ren YX, Li GX, Wang YD, Liu SD, Wang JD. MiR-646 inhibited cell proliferation and EMT-induced metastasis by targeting FOXK1 in gastric cancer. Br J Cancer. 2017;117:525-34.

14. Li H, Yue L, Xu H, Li N, Li J, Zhang Z, Zhao RC. Curcumin suppresses osteogenesis by inducing miR-126a-3p and vice suppressing the WNT/LRP6 pathway. Aging. 2019;11:6983-98.

15. Sebastian A, Hum NR, Murugesh DK, Hatsell S, Economides AN, Loots GG. Wnt co-receptors Lrp5 and Lrp6 differentially mediate Wnt3a signaling in osteoblasts. PLoS ONE. 2017;12:e0188264.

16. Wang J, Wang X, Li Z, Liu H, Teng Y. MicroRNA-183 suppresses retinoblastoma cell growth, invasion and migration by targeting LRP6. FEBS J. 2014;281:1355-65.

17. Yang C, Yao C, Tian R, Zhu Z, Zhao L, Li P, Chen H, Huang Y, Zhi E, Gong Y, Xue Y, Wang H, Yuan Q, He Z, Li Z. miR-202-3p regulates sertoli cell proliferation, synthesis function, and apoptosis by targeting LRP6 and cyclin D1 of Wnt/beta-catenin signaling. Mol Ther Nucl Acids. 2019;14:1-19.

18. Du C, Lv Z, Cao L, Ding C, Gyabaah OA, Xie H, Zhou L, Wu J, Zheng S. MiR126-3p suppresses tumor metastasis and angiogenesis of hepatocellular carcinoma by targeting LRP6 and PIK3R2. J Transl Med. 2014;12:259.

19. Chan JJ, Tay Y. Noncoding RNA: RNA regulatory networks in cancer. Int J Mol Sci. 2018;19(5):1310.

20. Zhong Y, Du Y, Yang X, Mo Y, Fan C, Xiong F, Ren D, Ye X, Li C, Wang Y, Wei F, Guo C, Wu X, Li X, Li Y, Li G, Zeng Z, Xiong W. Circular RNAs function as ceRNAs to regulate and control human cancer progression. Mol Cancer. 2018;17(1):79.

21. Zhao X, Cai Y, Xu J. Circular RNAs: biogenesis, mechanism, and function in human cancers. Int J Mol Sci. 2019;20(16):3926.

22. Geng $Y$, Jiang J, Wu C. Function and clinical significance of circRNAs in solid tumors. J Hematol Oncol. 2018;11(1):98

23. Xing L, Zhang L, Feng Y, Cui Z, Ding L. Downregulation of circular RNA hsa_circ_0001649 indicates poor prognosis for retinoblastoma and regulates cell proliferation and apoptosis via AKT/mTOR signaling pathway. Biomed Pharmacother. 2018;105:326-33.

24. Du S, Wang S, Zhang F, LV Y. SKP2, positively regulated by circ ODC1/miR$422 \mathrm{a}$ axis, promotes the proliferation of retinoblastoma. J Cell Biochem. 2020;121(1):322-31.

25. Galardi A, Colletti M, Di Paolo V, Vitullo P, Antonetti L, Russo I, Di Giannatale A. Exosomal MiRNAs in Pediatric Cancers. Int J Mol Sci. 2019;20(18):4600.

26. Szelenberger R, Kacprzak M, Saluk-Bijak J, Zielinska M, Bijak M. Plasma MicroRNA as a novel diagnostic. Clinica Chimica Acta Int J Clin Chem. 2019;499:98-107

27. Pan Y, Chen Y, Ma D, Ji Z, Cao F, Chen Z, Ning Y, Bai C. miR-646 is a key negative regulator of EGFR pathway in lung cancer. Exp Lung Res. 2016:42:286-95.

28. Dai H, Hou K, Cai Z, Zhou Q, Zhu S. Low-level miR-646 in colorectal cancer inhibits cell proliferation and migration by targeting NOB1 expression. Oncol Lett. 2017:14:6708-14. 
29. Li W, Liu M, Feng Y, Xu YF, Huang YF, Che JP, Wang GC, Yao XD, Zheng JH. Downregulated miR-646 in clear cell renal carcinoma correlated with tumour metastasis by targeting the nin one binding protein (NOB1). $\mathrm{Br}$ Cancer. 2014;111(6):1188-200.

30. Wang J, Shu H, Guo S. MiR-646 suppresses proliferation and metastasis of non-small cell lung cancer by repressing FGF2 and CCND2. Cancer Med. 2020. https://doi.org/10.1002/cam4.3062.

31. Li C, Li M, Xue Y. Downregulation of CircRNA CDR1 as specifically triggered low-dose Diosbulbin-B induced gastric cancer cell death by regulating miR-7-5p/REGgaRBa axis. Biomed Pharmacother. 2019;120:109462.

32. Raisch J, Cote-Biron A, Rivard N. A role for the WNT co-receptor LRP6 in pathogenesis and therapy of epithelial cancers. Cancers. 2019;11(8):1162.

33. Massink MP, Creton MA, Spanevello F, Fennis WM, Cune MS, Savelberg SM, Nijman IJ, Maurice RB, van den Boogaard MJ, van Haaften
G. Loss-of-function mutations in the WNT co-receptor LRP6 cause autosomal-dominant oligodontia. Am J Hum Genet. 2015;97:621-6.

34. Yan G, Li C, Zhao Y, Yue M, Wang L. Downregulation of microRNA6295p in colorectal cancer and prevention of the malignant phenotype by direct targeting of lowdensity lipoprotein receptor related protein 6. Int J Mol Med. 2019;44:1139-50.

35. Ma J, Lu W, Chen D, Xu B, Li Y. Role of Wnt co-receptor LRP6 in triple negative breast cancer cell migration and invasion. J Cell Biochem. 2017;118:2968-76.

\section{Publisher's Note}

Springer Nature remains neutral with regard to jurisdictional claims in published maps and institutional affiliations.
Ready to submit your research? Choose BMC and benefit from:

- fast, convenient online submission

- thorough peer review by experienced researchers in your field

- rapid publication on acceptance

- support for research data, including large and complex data types

- gold Open Access which fosters wider collaboration and increased citations

- maximum visibility for your research: over 100M website views per year

At BMC, research is always in progress.

Learn more biomedcentral.com/submissions 